\title{
Conductivity of silicon inversion layers: Comparison with and without an in-plane magnetic field
}

\author{
Yeekin Tsui, S. A. Vitkalov, and M. P. Sarachik \\ Physics Department, City College of the City University of New York, New York, New York 10031, USA \\ T. M. Klapwijk \\ Kavli Institute of Nanoscience, Delft University of Technology, Department of Applied Physics, 2628 CJ Delft, The Netherlands
}

(Received 8 July 2004; revised manuscript received 1 November 2004; published 17 March 2005)

\begin{abstract}
A detailed comparison is presented of the temperature dependence of the conductivity of dilute, strongly interacting electrons in two-dimensional silicon inversion layers in the metallic regime in the presence and in the absence of a magnetic field. We show explicitly and quantitatively that a magnetic field applied parallel to the plane of the electrons reduces the temperature dependence of the conductivity to near zero over a broad range of electron densities extending deep into the metallic regime where the high-field conductivity is on the order of $10 e^{2} / h$. The strong suppression (or "quenching") of the metallic behavior by a magnetic field sets an important constraint on theory.
\end{abstract}

DOI: 10.1103/PhysRevB.71.113308

PACS number(s): 73.40.Qv, 73.50.Jt, 71.30.+h

The conductivity of low density, strongly interacting electrons (or holes) in two dimensions (2D) increases with decreasing temperature above a critical electron density $n_{c}$ (or hole density $p_{c}$ ), raising the possibility that there exists an unexpected metallic phase and a metal-insulator transition in two dimensions. ${ }^{1}$ This behavior has been observed in many different 2D systems and is particularly pronounced in inversion layers in silicon metal-oxide semiconductor field-effect transistors (MOSFET's). The application of a magnetic field parallel to the plane of the electrons (or holes) has a dramatic effect, causing the conductivity to change by many orders of magnitude at low temperatures and low densities near $n_{c}$ $\left(p_{c}\right)$. In silicon MOSFET's, the conductivity decreases as the magnetic field is increased and then saturates to a value that is approximately constant. ${ }^{2,3}$ Other systems exhibit very similar behavior, with a conductivity that reaches a knee and then continues to decrease but with much smaller slope. ${ }^{4}$ Shubnikov-de Haas experiments have been performed that indicate that the electrons become fully polarized at or near the value of in-plane magnetic field that causes the saturation or knee observed in the magnetoconductivity. ${ }^{5-7}$ These intriguing and quite anomalous effects have been the subject of a great deal of interest and debate.

Although a number of studies have shown qualitatively that a magnetic field decreases the conductivity and suppresses the metallic behavior, ${ }^{2,8-10}$ there has been no systematic investigation of the temperature dependence in moderate and high magnetic field. The purpose of the present note is to demonstrate explicitly and quantitatively that the application of a magnetic field parallel to the plane of the electrons in silicon inversion layers sharply reduces the temperature dependence of the conductivity over a broad range extending to electron densities deep in the metallic regime where the conductivity at high field is on the order of $10 e^{2} / h$.

Data are presented for three silicon MOSFET's with mobilities $\mu$ at $4.2 \mathrm{~K}$ of $\approx 30000 \mathrm{~V} /\left(\mathrm{cm}^{2} \mathrm{~s}\right.$ ) (sample No. 1) and $20000 \mathrm{~V} /\left(\mathrm{cm}^{2} \mathrm{~s}\right)$ (sample Nos. 2 and 3). Contact resistances were minimized by using a split-gate geometry, which allows a higher electron density in the vicinity of the contacts than in the $2 \mathrm{D}$ system under investigation. The resistance was measured in a ${ }^{3} \mathrm{He}$ Oxford Heliox system as a function of temperature in zero field and in a parallel field of $10 \mathrm{~T}$ by standard four-probe ac techniques using currents in the linear regime, typically below $5 \mathrm{nA}$, at frequency $3 \mathrm{~Hz}$. Metallic temperature dependence was found in zero field for all samples at electron densities above $n_{c} \approx 0.9 \times 10^{11} \mathrm{~cm}^{-2}$.

The conductivity of a silicon MOSFET sample in the absence of magnetic field is shown as a function of temperature for eight different electron densities in Fig. 1(a); Fig. 1(b) shows the conductivity for the same electron densities in a magnetic field of $10 \mathrm{~T}$ applied parallel to the electron plane. The temperature dependence in the absence of a field is strongly suppressed by an in-plane magnetic field of $10 \mathrm{~T}$. Similar results were obtained for the two other samples studied. It should be noted that the conductivity is near or at its high-field, saturated value in $10 \mathrm{~T}$ for all the densities shown.

In order to demonstrate the effect of high in-plane magnetic fields, we need to obtain an estimate of the temperature dependence with and without magnetic field. The conductivity is not a linear function of temperature. However, there is a range of temperature over which linear fits have been applied by several groups ${ }^{11-14}$ in order to compare data with the theory of Zala et al. ${ }^{15}$ which predicts linear behavior over a restricted range of temperature. Using this procedure, the slopes of the conductivity curves $d \sigma / d T$ were determined in zero field and in high magnetic field, as illustrated in Fig. 2 for two different electron densities.

We now examine how the temperature dependence of the conductivity evolves as the magnetic field is increased from zero to a value high enough that the conductivity has reached its high-field saturated value where the electron spins are completely aligned. For a constant electron density $n=1.64$ $\times 10^{11} \mathrm{~cm}^{-2}$, the closed symbols of Fig. 3 are the slopes $d \sigma(H) / d T$ of the $\sigma$ versus $T$ curves for several values of in-plane magnetic field. Although the $\sigma$ versus $T$ curves at finite field exhibit some detailed structure, ${ }^{16}$ these are small effects that do not affect the overall behavior in a substantial way. In order to demonstrate that detailed structure and non- 

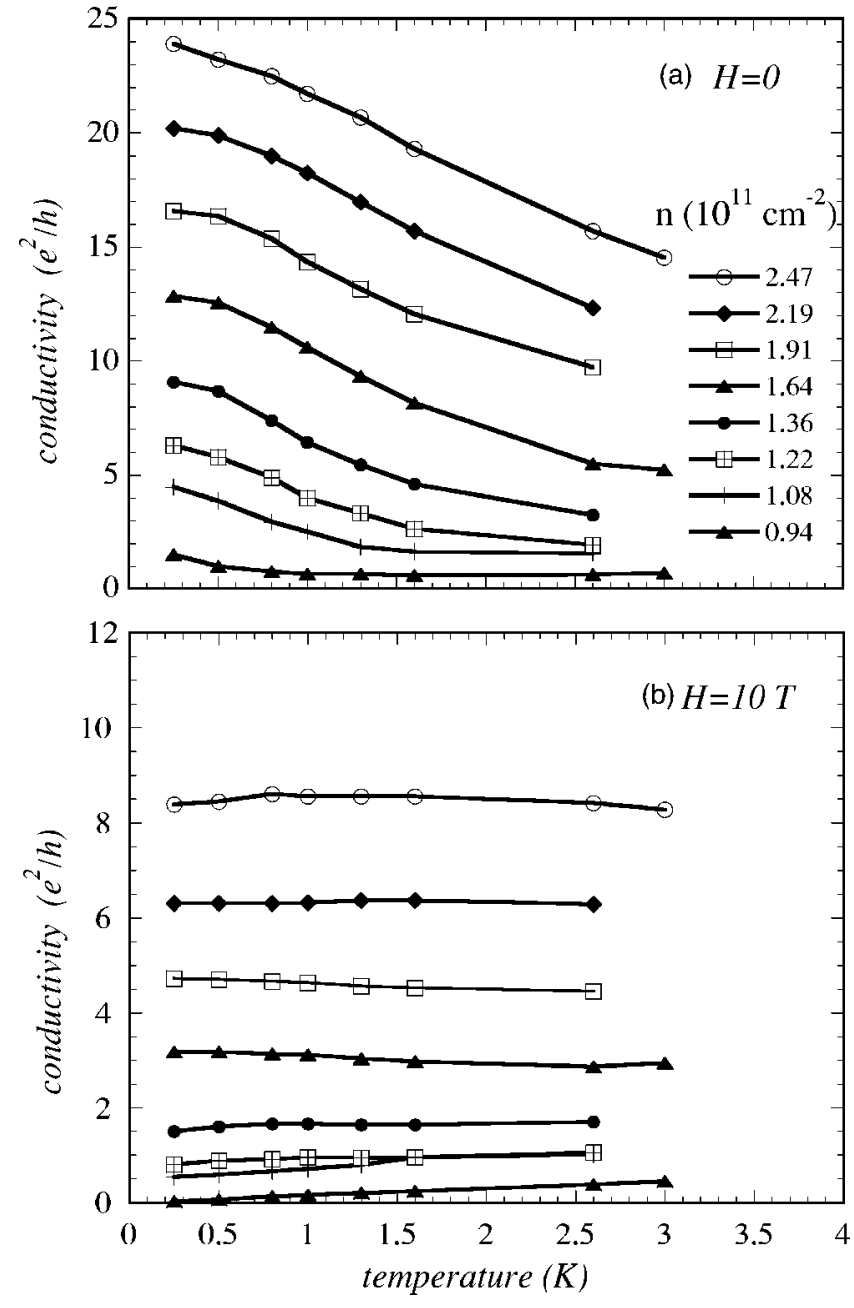

FIG. 1. Conductivity of silicon MOSFET sample No. 2 as a function of temperature for different electron densities, as labeled: (a) in the absence of external magnetic field; (b) in a field of $10 \mathrm{~T}$ applied parallel to the plane. Similar results were obtained for sample Nos. 1 and 3.

linear behavior are unimportant to our conclusions, the open symbols in Fig. 3 show for comparison the quantity $\Delta \sigma / \Delta T$ obtained from the slope of a line drawn between data points at 1.35 and $0.27 \mathrm{~K}$. The main features of the curve are unaltered. The (negative) slope changes rapidly with increasing in-plane magnetic field and assymptotically approaches a value near zero as the field approaches the value required to saturate the conductivity and align the spins.

The behavior illustrated in Fig. 3 obtains over a broad range of electron densities deep into the metallic phase, where the conductivity is 10 to 20 times the quantum unit of conductance. Figure 4 shows the ratio of the slope in a high in-plane field of $10 \mathrm{~T}$ to the slope in zero field $d \sigma(H$ $=10 \mathrm{~T}) / d T /[d \sigma(0) / d T]$, plotted as a function of electron density. As indicated by the dashed horizontal lines, the ratio does not exceed \pm 0.1 and is near zero over the range of densities studied, from $n=1.22 \times 10^{11} \mathrm{~cm}^{-2}$ to 2.5 $\times 10^{11} \mathrm{~cm}^{-2}$. The temperature dependence in high in-plane magnetic field is weak but finite. We attribute this to remanent, weak effects that become dominant when the field has
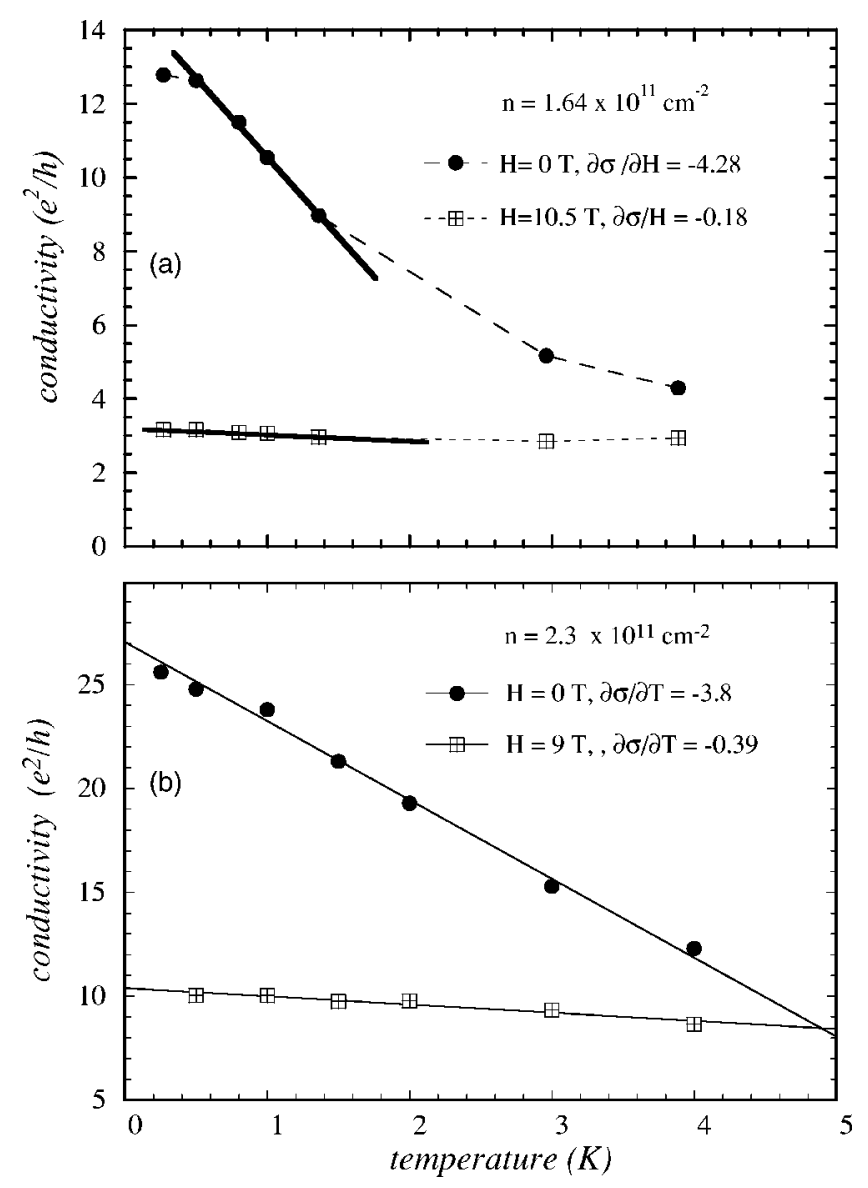

FIG. 2. For two electron densities, the lines illustrate the procedure used to determine the slope $d \sigma / d T$ plotted in Fig. 4. Note that the range over which the slope is approximately constant broadens as the electron density is increased. Data shown for sample No. 3.

suppressed the strong zero-field dependence on temperature.

Many theories have been proposed to account for the interesting behavior of two-dimensional systems of electrons such as silicon MOSFET's. Temperature-dependent screening in a Fermi gas has been suggested by many ${ }^{17}$ as the source of the temperature dependence of the conductivity. A number of other theoretical scenarios have been advanced, including percolation in an inhomogeneous system composed of metallic and insulating regions, a Wigner crystal or glass, ferromagnetism, superconductivity, a spin glass, and electron glass. ${ }^{1}$ Complete suppression of the zero-field temperature dependence by in-plane magnetic field has been found in numerical studies. ${ }^{18}$ It is also predicted by the theory of Spivak and Kivelson, ${ }^{19}$ which considers phase separation and intermediate phases between the Fermi liquid and the Wigner crystal.

In summary, data are reported for inversion layers in high mobility silicon MOSFET's that demonstrate quantitatively that an in-plane magnetic field suppresses the metallic temperature dependence of the conductivity observed in the absence of magnetic field. The metallic behavior is strongly suppressed (or "quenched") over a broad range of densities extending deep into the metallic regime where the high-field conductivity is ten times the quantum unit of conductance. 


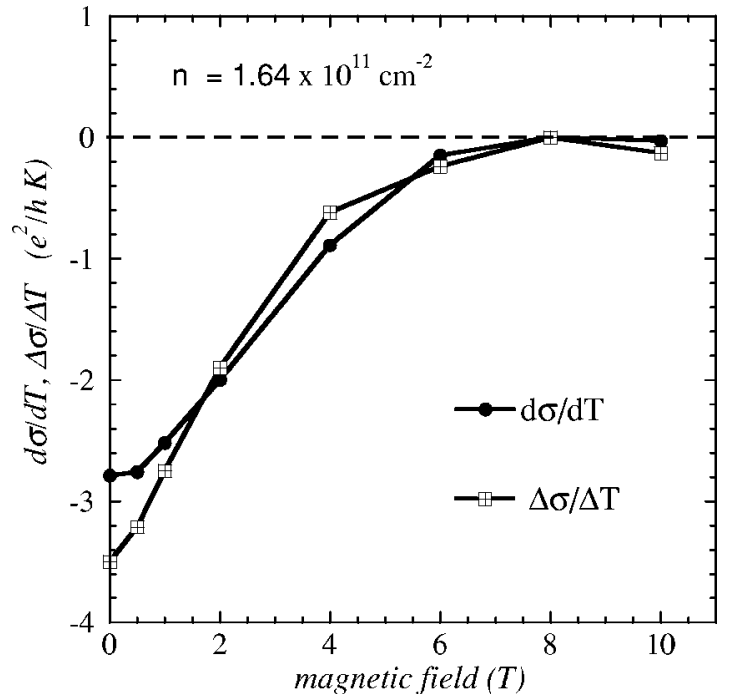

FIG. 3. The closed symbols denote the slope $d \sigma / d T$ versus inplane magnetic field $H$ for silicon MOSFET sample No. 2 at electron density $1.64 \times 10^{11} \mathrm{~cm}^{-2}$. The open symbols denote $\Delta \sigma / \Delta T$ calculated for the temperature interval 0.27 to $1.35 \mathrm{~K}$.

This is a robust and central feature of these two-dimensional systems that must be accounted for by any theory. We suggest that the effect of magnetic field is key to understanding the enigmatic zero-field behavior of strongly interacting twodimensional systems.

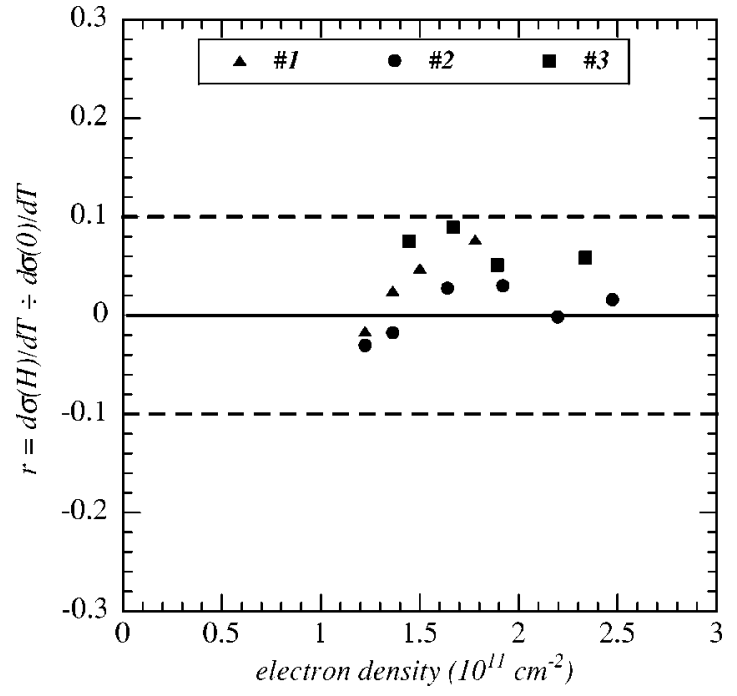

FIG. 4. The ratio $r=d \sigma\left(H_{\text {sat }}\right) / d T /[d \sigma(0) / d T]$ versus electron density for three samples, as labeled.

We thank Boris Spivak and Steve Kivelson for stimulating this paper and for numerous discussions. This work was supported by Grant No. DOE-FG02-84-ER45153 and NSF Grant No. DMR-0129581. S.A.V. acknowledges support from Grant No. NSF DMR 0349049.
${ }^{1}$ For reviews, see E. Abrahams, S. V. Kravchenko, and M. P. Sarachik, Rev. Mod. Phys. 73, 251 (2001); S. V. Kravchenko and M. P. Sarachik, Rep. Prog. Phys. 67, 1 (2004); V. M. Pudalov, cond-mat/0405315 (unpublished); A. A. Shashkin, cond-mat/ 0405556 (unpublished).

${ }^{2}$ D. Simonian, S. V. Kravchenko, M. P. Sarachik, and V. M. Pudalov, Phys. Rev. Lett. 79, 2304 (1997).

${ }^{3}$ V. M. Pudalov, G. Brunthaler, A. Prinz, and G. Bauer, Pis'ma Zh. Eksp. Teor. Fiz. 65, 887 (1997) [JETP Lett. 65, 932 (1997)].

${ }^{4}$ J. Yoon, C. C. Li, D. Shahar, D. C. Tsui, and M. Shayegan, Phys. Rev. Lett. 84, 4421 (2000); R. Pillarisetty, H. Noh, E. Tutuc, E. P. De Poortere, D. C. Tsui, and M. Shayegan, ibid. 90, 226801 (2003).

${ }^{5}$ T. Okamoto, K. Hosoya, S. Kawaji, and A. Yagi, Phys. Rev. Lett. 82, 3875 (1999).

${ }^{6}$ S. A. Vitkalov, H. Zheng, K. M. Mertes, M. P. Sarachik, and T. M. Klapwijk, Phys. Rev. Lett. 85, 2164 (2000); S. A. Vitkalov, M. P. Sarachik, and T. M. Klapwijk, Phys. Rev. B 64, 073101 (2000)

${ }^{7}$ E. Tutuc, E. P. De Poortere, S. J. Papadakis, and M. Shayegan, Phys. Rev. Lett. 86, 2858 (2001).

${ }^{8}$ V. T. Dolgopolov, G. V. Kravchenko, A. A. Shashkin, and S. V. Kravchenko, JETP Lett. 55, 733 (1992).

${ }^{9}$ K. M. Mertes, H. Zheng, S. A. Vitkalov, M. P. Sarachik, and T.
M. Klapwijk, Phys. Rev. B 63, 041101(R) (2001).

${ }^{10}$ A. A. Shashkin, S. V. Kravchenko, and T. M. Klapwijk, Phys. Rev. Lett. 87, 266402 (2001).

${ }^{11}$ A. A. Shashkin, S. V. Kravchenko, V. T. Dolgopolov, and T. M. Klapwijk, Phys. Rev. B 66, 073303 (2002).

${ }^{12}$ Y. Y. Proskuryakov, A. K. Savchenko, S. S. Safonov, M. Pepper, M. Y. Simmons, and D. A. Ritchie, Phys. Rev. Lett. 89, 076406 (2002).

${ }^{13}$ S. A. Vitkalov, K. James, B. N. Narozhny, M. P. Sarachik, and T. M. Klapwijk, Phys. Rev. B 67, 113310 (2003).

${ }^{14}$ V. M. Pudalov, M. E. Gershenson, H. Kojima, G. Brunthaler, A. Prinz, and G. Bauer, Phys. Rev. Lett. 91, 126403 (2003).

${ }^{15}$ G. Zala, B. N. Narozhny, and I. L. Aleiner, Phys. Rev. B 64, 214204 (2001); 65, 020201(R) (2002).

${ }^{16}$ Yeekin Tsui, S. A. Vitkalov, and M. P. Sarachik (unpublished).

${ }^{17}$ See, for examples, T. Ando, A. B. Fowler, and F. Stern, Rev. Mod. Phys. 54, 437 (1982); S. Das Sarma, Phys. Rev. B 33, 5401 (1986); A. Gold and V. T. Dolgopolov, ibid. 33, 1076 (1986); S. Das Sarma and E. H. Hwang, Phys. Rev. Lett. 83, 164 (1999); I. F. Herbut, Phys. Rev. B 63, 113102 (2001).

${ }^{18}$ See, for example, P. J. H. Denteneer and R. T. Scalettar, Phys. Rev. Lett. 90, 246401 (2003).

${ }^{19}$ B. Spivak, Phys. Rev. B 67, 125205 (2002); B. Spivak and S. A. Kivelson, ibid. 70, 155114 (2004). 